\title{
Die Praxis sollte das Ergebnis des Nachdenkens sein.
}

\author{
Hermann Hesse
}

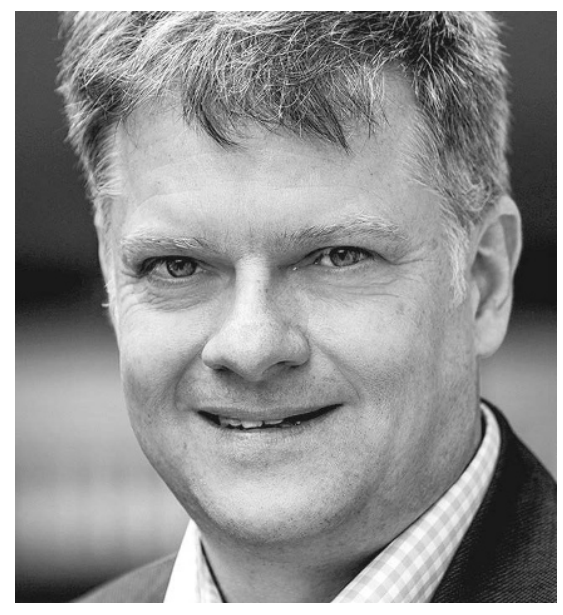

Zwar wurden durch die Datenschutz-Grundverordnung (DSGVO) nur wenige Datenschutz-Anforderungen des damaligen Bundesdatenschutzgesetzes substantiell geändert oder erweitert. Aber nicht zuletzt die inzwischen auch in Deutschland Aufsehen erregenden Bußgelder der Aufsichtsbehörden haben zu einer deutlich intensiveren Beschäftigung mit konkreten Praxisfragen der Umsetzung geführt.

So stellt die an die Stelle der Vorabkontrolle getretene Datenschutz-Folgenabschätzung viele Unternehmen und Behörden vor Herausforderungen. Wann ist sie erforderlich? Wie genau ist dabei vorzugehen? Welche Maßnahmen sind anschließend zu ergreifen? Nägele, Petrlic und Schemmel widmen sich diesem Themenkomplex aus drei unterschiedlichen Perspektiven: der betrieblichen Praxis, der Lehre und Forschung und der einer Aufsichtsbehörde.

Eine besondere Anforderung stellt die Meldefrist von 72 Stunden bei einem „Data breach" nach Art. 33 DSGVO in der Praxis dar - sie läuft auch über Sonn- und Feiertage. Dabei kann es schnell knapp werden. Labusga untersucht in seinem Beitrag, wie Fristbeginn und Fristende rechtskonform zu bestimmen sind.

Wie gut Unternehmen tatsächlich dem nun wahrlich nicht neuen Recht auf Auskunft nachkommen, untersuchten Weik und Dieh/ in der ersten Jahreshälfte in einer Praxisstudie. Die Ergebnisse sind - wie zu befürchten - durchwachsen. Auch hier besteht offenbar auch im 43. Jahr des Datenschutzrechts noch Nachholbedarf

Wie schwierig es ist, komplexe technische Lösungen datenschutzrechtlich zu bewerten und praxisgeeignete Vorgehensweisen zu deren Einsatz zu empfehlen, zeigt die aktuelle Diskussion um Videokonferenzsysteme. Gerling, Gerling, Hessel und Petrlic beziehen aus einer technischen Perspektive Position zu diesbezüglichen Stellungnahmen der Datenschutz-Aufsichtsbehörden. Und auch der immer wieder nicht zuletzt von Aufsichtsbehörden geforderte Einsatz einer Ende-zu-Ende-Verschlüsselung von E-Mails stößt auf praktische Schwierigkeiten, wie Schleipfer in seinem Forums-Beitrag hervorhebt.

Erste Erfahrungen mit einem interessanten Ansatz zur Umsetzung von „Privacy by Design" stellen Kus und Sohr vor. Sie verwenden die von Microsoft für die Bedrohungsmodellierung entwickelte STRIDE-Methode, die sich bei Risikobewertungen bereits in der Praxis bewährt hat.

Die praktischen Umsetzungsfragen wirken bis in die Gesetzgebung hinein. Das gilt insbesondere für die Regulierung der zunehmenden Digitalisierung. Schrahe und Städter untersuchen in ihrem Beitrag die praktischen Implikationen und Regulierungslücken des Digitale-Versorgungs-Gesetzes (DVG) und des PatientendatenSchutz-Gesetzes. Vor allem die Frage einer geeigneten Pseudonymisierung von Patientendaten für die Nutzung in der Forschung ist ein besonders kritischer Punkt, hängt davon doch unmittelbar das mit der Verarbeitung verbundene Risiko für die Betroffenen ab.

\section{Dirk Fox}

